Pacific

Journal of

Mathematics

SYMPLECTIC FLOER HOMOLOGY AND THE MAPPING CLASS GROUP

Paul Seidel 


\title{
SYMPLECTIC FLOER HOMOLOGY AND THE MAPPING CLASS GROUP
}

\author{
PAUl SEIDEL
}

\begin{abstract}
We consider symplectic Floer homology in the lowest nontrivial dimension, that is to say, for area-preserving diffeomorphisms of surfaces. Particular attention is paid to the quantum cap product.
\end{abstract}

\section{Introduction}

Let $\Gamma=\pi_{0}\left(\operatorname{Diff}^{+}(M)\right)$ be the mapping class group of a closed connected oriented surface $M$ of genus $\geq 2$. Pick an everywhere positive two-form $\omega$ on $M$. A theorem of Moser says that each $g \in \Gamma$ admits representatives which preserve $\omega$. These are automorphisms of the two-dimensional symplectic manifold $(M, \omega)$, and the techniques of symplectic topology can be applied to them. We will use symplectic representatives $\phi$ which satisfy a certain additional monotonicity property. The benefit is that the Floer homology $H F_{*}(\phi)$, which is a finite-dimensional $\mathbb{Z} / 2$-graded vector space over the field $\mathbb{Z} / 2$, is independent of the choice of $\phi$, hence an invariant of $g$; we denote it by $H F_{*}(g)$. There is an additional multiplicative structure, the quantum cap product

$$
*: H^{*}(M ; \mathbb{Z} / 2) \otimes H F_{*}(\phi) \rightarrow H F_{*}(\phi) ;
$$

this is again independent of the choice of representative $\phi$, thus equipping $H F_{*}(g)$ with the structure of a $\mathbb{Z} / 2$-graded $H^{*}(M ; \mathbb{Z} / 2)$-module. The most familiar case is when $g=e: H F_{*}(e) \cong H_{*}(M ; \mathbb{Z} / 2)$, and $*$ reduces to the ordinary cap product (this was announced in [9] and [8]; a detailed proof, in greater generality than envisaged by the earlier approaches, has appeared in [6]). In particular, the quantum cap action of $H^{2}(M ; \mathbb{Z} / 2) \cong \mathbb{Z} / 2$ on $H F_{*}(e)$ is nonzero. Our first result is that this characterizes the trivial mapping class.

Theorem 1. For all $g \neq e$ in $\Gamma$, the quantum cap action of $H^{2}(M ; \mathbb{Z} / 2)$ on $H F_{*}(g)$ is zero.

Floer homology does not distinguish between arbitrary mapping classes: There are cases when $H F_{*}\left(g_{1}\right) \cong H F_{*}\left(g_{2}\right)$ as $H^{*}(M ; \mathbb{Z} / 2)$-modules and where $g_{1}, g_{2}$ are not even conjugate. An easy source of such examples are 
fixed point free diffeomorphisms of finite order, since their Floer homology is always zero.

Theorem 2. Suppose that $a \in H^{1}(M ; \mathbb{Z} / 2)$ is a class whose quantum cap action on $H F_{*}(g)$ is nonzero. Then there is an $l: S^{1} \rightarrow M$ with $g(l) \simeq l$ and $\langle a,[l]\rangle=1$.

Here $\simeq$ denotes the relation of free homotopy between loops in $M$. The same examples as before can be used to see that the converse to Theorem 2 is false. By this we mean that one can have $g(l) \simeq l$ with $[l] \neq 0$ in $H_{1}(M ; \mathbb{Z} / 2)$, and the action of $H^{1}(M ; \mathbb{Z} / 2)$ on $H F_{*}(g)$ can still be zero.

The strategy of proof is to consider a certain infinite (not usually Galois or connected) covering $p_{\phi}: \tilde{M}_{\phi} \rightarrow M$ canonically associated to $\phi \in \operatorname{Diff}^{+}(M)$. One can introduce a refined quantum cap product, in which the cohomology of this covering replaces that of $M$. More precisely:

Lemma 3. Let $\phi$ be a monotone symplectic automorphism of $(M, \omega)$. Then there is a map $\tilde{*}$ fitting into a commutative diagram

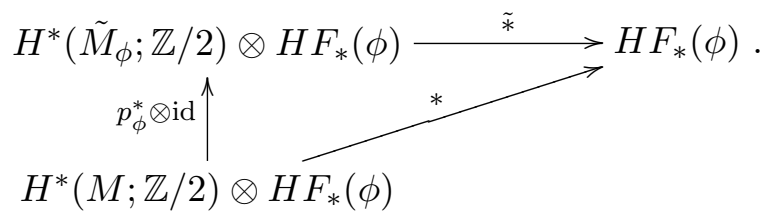

In particular, if the quantum cap action of $a \in H^{*}(M ; \mathbb{Z} / 2)$ is nonzero, $p_{\phi}^{*}(a)$ must be nonzero.

This, together with elementary topological arguments, yields the theorems above. To round off the discussion, we should mention that Floer homology can also be defined with integer coefficients. Our arguments carry over without any changes, and in the case of Theorem 2 this leads to a slightly better control over the homology class of $l$.

\section{Three topological preliminaries.}

(a) Let $\phi \in \operatorname{Diff}^{+}(M)$. The definition of the covering $p_{\phi}: \tilde{M}_{\phi} \rightarrow M$ is that points of $\tilde{M}_{\phi}$ are pairs $(x,[c])$, where $x \in M, c:[0 ; 1] \rightarrow M$ is a path from $c(0)=\phi(x)$ to $c(1)=x$, and $[c]$ is the homotopy class of $c$ rel endpoints. Equivalently, after choosing a hyperbolic metric on $M$, one can describe $\tilde{M}_{\phi}$ as the space of all geodesic paths $c:[0 ; 1] \rightarrow M$ such that $c(0)=\phi(c(1))$. There is a canonical lift of $\phi$ to a diffeomorphism $\tilde{\phi}: \tilde{M}_{\phi} \rightarrow \tilde{M}_{\phi}, \tilde{\phi}(x,[c])=(\phi(x),[\phi(c)])$; and it is not difficult to see that $\tilde{\phi}$ is homotopic to the identity map. In particular, if a loop $l: S^{1} \rightarrow M$ can be lifted to $\tilde{M}_{\phi}$ then $\phi(l) \simeq l$; and the converse is also true. This has an immediate consequence: 
Lemma 4. Let $a \in H^{1}(M ; \mathbb{Z} / 2)$ be a class such that $p_{\phi}^{*}(a) \neq 0$. Then there is a loop $l$ such that $\phi(l) \simeq l$ and $\langle a,[l]\rangle=1$.

Now suppose that $\tilde{M}_{\phi}$ has a connected component which is compact, so that the restriction of $p_{\phi}$ to it is a covering of finite order. Then every loop $l$ has an iterate $l^{k}, k \geq 1$, which lifts to $\tilde{M}_{\phi}$, so that $\phi(l)^{k} \simeq l^{k}$. As one can see by looking at the unique geodesic representatives, this implies $\phi(l) \simeq l$. From that one can deduce in various ways that $[\phi] \in \Gamma$ is trivial, for instance: Since lengths of closed geodesics form a set of coordinates on the Teichmüller space $\mathcal{T}$ of $M[\mathbf{3}$, Exposé 7 , Théorème 4], it follows that the class $g=[\phi] \in \Gamma$ acts trivially on $\mathcal{T}$. In other words, every hyperbolic metric on $M$ admits an isometry lying in $g$. This means that $g=e$, or else that $M$ is of genus two and $\phi$ is isotopic to a hyperelliptic involution. However, hyperelliptic involutions act as -1 on $H_{1}(M ; \mathbb{Z})$, hence they can not satisfy $\phi(l) \simeq l$ for homologically nontrivial $l$. We have therefore proved:

Lemma 5. If $H^{2}\left(\tilde{M}_{\phi} ; \mathbb{Z} / 2\right)$ is nonzero, $[\phi] \in \Gamma$ is the identity class.

(b) For $\phi$ in the group $\operatorname{Symp}(M, \omega)$ of symplectic automorphisms, consider its mapping torus $T_{\phi}=(\mathbb{R} \times M) /(t+1, x) \sim(t, \phi(x))$, which is a threemanifold fibered over $S^{1}$. The pullback of $\omega$ to $\mathbb{R} \times M$ descends to a closed two-form $\omega_{\phi} \in \Omega^{2}\left(T_{\phi}\right)$. The tangent bundle along the fibres of $T_{\phi} \rightarrow S^{1}$ is an oriented real two-plane bundle, whose Euler class we denote by $c_{\phi} \in$ $H^{2}\left(T_{\phi} ; \mathbb{R}\right)$. Now $H^{*}\left(T_{\phi} ; \mathbb{R}\right)$ fits into an exact sequence

$$
\cdots \rightarrow H^{1}(M ; \mathbb{R}) \stackrel{\text { id }-\phi^{*}}{\longrightarrow} H^{1}(M ; \mathbb{R}) \stackrel{d}{\rightarrow} H^{2}\left(T_{\phi} ; \mathbb{R}\right) \rightarrow H^{2}(M ; \mathbb{R}),
$$

where the last map is restriction to the fibre. Since the difference $\left[\omega_{\phi}\right]-$ $\left(\int_{M} \omega / \chi(M)\right) c_{\phi}$ vanishes when restricted to a fibre, it is of the form $d(m(\phi))$ for some unique $m(\phi) \in H^{1}(M ; \mathbb{R}) / \mathrm{im}\left(\mathrm{id}-\phi^{*}\right)$. A computation shows the following:

Lemma 6. Let $\left(\psi_{t}\right)_{0 \leq t \leq 1}$ be an isotopy in $\operatorname{Symp}(M, \omega)$ with $\psi_{0}=\mathrm{id}$, and $c \in H^{1}(M ; \mathbb{R})$ its Calabi invariant. Then for any $\phi \in \operatorname{Symp}(M, \omega)$ one has $m\left(\phi \circ \psi_{1}\right)=m(\phi)+c$.

Call $\phi$ monotone if $m(\phi)=0$. These maps form a closed subspace (not subgroup) $\operatorname{Symp}^{m}(M, \omega) \subset \operatorname{Symp}(M, \omega)$, and using Lemma 6 one can show that this is a deformation retract of $\operatorname{Symp}(M, \omega)$. By combining this with Moser's theorem [7] that $\operatorname{Symp}(M, \omega) \hookrightarrow \operatorname{Diff}^{+}(M)$ is a homotopy equivalence, and the Earle-Eells theorem [2] that the connected components of $\operatorname{Diff}^{+}(M)$ are contractible, one gets:

Lemma 7. The inclusion $\operatorname{Symp}^{m}(M, \omega) \hookrightarrow \operatorname{Diff}^{+}(M)$ induces an isomorphism $\pi_{0}\left(\operatorname{Symp}^{m}(M, \omega)\right) \cong \Gamma$. Moreover, each connected component of $\operatorname{Symp}^{m}(M, \omega)$ is contractible. 
(c) Choose a Morse function $f$ on $M$, and a Riemannian metric such that the gradient flow of $f$ is Morse-Smale. The Morse cochain complex $C M^{*}(f)$ is the graded vector space over $\mathbb{Z} / 2$ freely generated by the critical points of $f$, with the degree of a generator $\langle x\rangle$ given by its Morse index $\mu(x)$. For $\mu\left(x_{+}\right)=\mu\left(x_{-}\right)+1$, let $l\left(x_{-}, x_{+}\right) \in \mathbb{Z} / 2$ be the number modulo 2 of flow lines of $\nabla f$ going from $x_{-}$to $x_{+}$. One defines a differential $\delta_{f}: C M^{*}(f) \rightarrow C M^{*+1}(f)$ by $\delta_{f}\left\langle x_{-}\right\rangle=\sum_{x_{+}} l\left(x_{-}, x_{+}\right)\left\langle x_{+}\right\rangle$. The cohomology $H\left(C M^{*}(f), \delta_{f}\right)$ is canonically isomorphic to $H^{*}(M ; \mathbb{Z} / 2)$; see $[\mathbf{1 1}]$ for a proof.

Now let $p: \tilde{M} \rightarrow M$ be a covering, and $\tilde{f}=f \circ p$. Consider the graded vector space $C M^{*}(\tilde{f})$ whose elements are (possibly infinite) formal sums of critical points of $\tilde{f}$, with $\mathbb{Z} / 2$ coefficients. Counting flow lines of $\nabla \tilde{f}$ with respect to the pullback metric yields numbers $\tilde{l}\left(\tilde{x}_{-}, \tilde{x}_{+}\right) \in \mathbb{Z} / 2$ for any two critical points $\tilde{x}_{-}, \tilde{x}_{+}$of $\tilde{f}$ with $\mu\left(\tilde{x}_{+}\right)=\mu\left(\tilde{x}_{-}\right)+1$, and from those numbers one constructs a differential $\delta_{\tilde{f}}$ as before. The argument from [11] can be adapted to show that there is a canonical isomorphism

$$
H\left(C M^{*}(\tilde{f}), \delta_{\tilde{f}}\right) \cong H^{*}(\tilde{M} ; \mathbb{Z} / 2)
$$

Since any flow line of $\nabla \tilde{f}$ is the lift of a flow line of $\nabla f$, one finds that for any critical points $x_{-}, x_{+}$of $f$ and any $\tilde{x}_{+} \in p^{-1}\left(x_{+}\right)$,

$$
l\left(x_{-}, x_{+}\right)=\sum_{p\left(\tilde{x}_{-}\right)=x_{-}} \tilde{l}\left(\tilde{x}_{-}, \tilde{x}_{+}\right) .
$$

This implies that the homomorphism $p^{*}: C M^{*}(f) \rightarrow C M^{*}(\tilde{f})$ which takes any critical point to the sum of its preimages is a chain map. Via the canonical isomorphisms, its induced map can be identified with the usual pullback $p^{*}: H^{*}(M ; \mathbb{Z} / 2) \rightarrow H^{*}(\tilde{M} ; \mathbb{Z} / 2)$ on cohomology.

\section{Floer homology.}

Fix $\phi \in \operatorname{Symp}^{m}(M, \omega)$. Let $\Lambda_{\phi}=\left\{y \in C^{\infty}(\mathbb{R}, M) \mid y(t)=\phi(y(t+1))\right\}$ be the twisted free loop space, which is also the space of sections of $T_{\phi} \rightarrow S^{1}$. The action form is the closed one-form $\alpha_{\phi}$ on $\Lambda_{\phi}$ defined by

$$
\alpha_{\phi}(y) Y=\int_{0}^{1} \omega(d y / d t, Y(t)) d t
$$

The main difference between our exposition and the ones in the existing literature, such as $[\mathbf{1}]$, is that we will admit a larger-than-usual class of perturbations of this one-form. 
Definition 8. $\mathcal{B}$ is the space of smooth families $b=\left(b_{t}\right)_{t \in \mathbb{R}}$ of closed oneforms on $M$ satisfying $b_{t+1}=\phi^{*} b_{t}$ and

$$
\int_{0}^{1}\left[b_{t}\right] d t \in \operatorname{im}\left(\mathrm{id}-\phi^{*}\right) \subset H^{1}(M ; \mathbb{R}) .
$$

A condition equivalent to (2) is that there should be closed one-forms $a_{t}$ and functions $H_{t}$ on $M$ such that $a_{t+1}=\phi^{*} a_{t}, H_{t+1}=\phi^{*} H_{t}$, and $b_{t}=$ $-\partial a_{t} / \partial t+d H_{t}$. For a more intrinsic formulation consider the closed twoform on $T_{\phi}$ whose pullback to $\mathbb{R} \times M$ is $d t \wedge b_{t}$. Then (2) is equivalent to saying that this two-form is exact; in fact it is $-d\left(a_{t}+H_{t} d t\right)$. Define the $b$-perturbed action form to be

$$
\alpha_{\phi, b}(y) Y=\alpha_{\phi}(y) Y+\int_{0}^{1} b_{t}(Y(t)) d t .
$$

The perturbation term which we have added is exact, since it is the derivative of the function $y \mapsto \int_{0}^{1} a_{t}(d y / d t)+H_{t}(y(t)) d t$ on $\Lambda_{\phi}$. Hence $\left[\alpha_{\phi, b}\right]=\left[\alpha_{\phi}\right] \in$ $H^{1}\left(\Lambda_{\phi} ; \mathbb{R}\right)$. The set $\mathcal{Z}(b)$ of zeros of $\alpha_{\phi, b}$ consists of the solutions $y \in \Lambda_{\phi}$ of

$$
d y / d t=X_{b, t}(y(t))
$$

where $X_{b, t}$ is the symplectic vector field dual to $b_{t}$. There is an open dense subset $\mathcal{B}^{\text {reg }} \subset \mathcal{B}$ such that if $b$ lies in it, all solutions of (3) are nondegenerate. Nondegeneracy means that the map $L_{y}: T M_{y(0)} \rightarrow T M_{y(1)}$ obtained by linearizing (3) near $y$ satisfies $\operatorname{det}\left(\mathrm{id}-D \phi_{y(1)} \circ L_{y}\right) \neq 0$. One then defines $C F^{*}(\phi, b)$ to be the vector space over $\mathbb{Z} / 2$ freely generated by the finite set $\mathcal{Z}(b)$, with a $\mathbb{Z} / 2$-grading given by the sign of the above-mentioned determinant.

Let $\mathcal{J}$ be the space of smooth families $J=\left(J_{t}\right)_{t \in \mathbb{R}}$ of $\omega$-compatible almost complex structures on $M$ such that $J_{t+1}=\phi^{*} J_{t}$. For $b \in \mathcal{B}^{\text {reg }}, J \in \mathcal{J}$, and $y_{-}, y_{+} \in \mathcal{Z}(b)$, let $\mathcal{M}\left(y_{-}, y_{+}, b, J\right)$ be the set of maps $u: \mathbb{R}^{2} \rightarrow M$ which satisfy

$$
\left\{\begin{array}{l}
u(s, t)=\phi(u(s, t+1)), \\
\lim _{s \rightarrow \pm \infty} u(s, \cdot)=y_{ \pm}, \\
\partial u / \partial s+J_{t}(u)\left(\partial u / \partial t-X_{b, t}(u)\right)=0 .
\end{array}\right.
$$

To each such map is associated a Fredholm operator $D_{u}$ which linearizes (4) in suitable Sobolev spaces. We write $\mathcal{M}_{k}\left(y_{-}, y_{+}, b, J\right)$ for the subset of those $u$ with index $\left(D_{u}\right)=k \in \mathbb{Z}$.

Lemma 9. The energy

$$
E(u)=\int_{\mathbb{R} \times[0 ; 1]}\left|\frac{\partial u}{\partial s}\right|_{J_{t}}^{2}
$$

is constant on each $\mathcal{M}_{k}\left(y_{-}, y_{+}, b, J\right)$. 
Proof. Take $u, v \in \mathcal{M}_{k}\left(y_{-}, y_{+}, b, J\right)$ and consider them as maps $\mathbb{R} \rightarrow \Lambda_{\phi}$, $s \mapsto u(s, \cdot)$ respectively $v(s, \cdot)$, with the same asymptotic behaviour. Because of the gradient flow interpretation of (4), the energy of each is equal to minus the integral of $\alpha_{\phi, b}$ along it. Gluing $u, v$ together at the ends yields a map $w=u \# \bar{v}: S^{1} \rightarrow \Lambda_{\phi}$, unique up to homotopy, such that $E(u)-E(v)=$ $-\int_{S^{1}} w^{*} \alpha_{\phi, b}=-\int_{S^{1}} w^{*} \alpha_{\phi}$. One may view $w$ as a map $S^{1} \times S^{1} \rightarrow T_{\phi}$, and rewrite the last equality as

$$
E(u)-E(v)=\int_{S^{1} \times S^{1}} w^{*} \omega_{\phi}
$$

The fact that index $\left(D_{u}\right)=\operatorname{index}\left(D_{v}\right)=k$, together with a standard gluing theorem for the index, shows that $w^{*} c_{\phi} \in H^{2}\left(S^{1} \times S^{1}\right)$ is zero. Since $\phi$ is monotone this implies that the right hand side of (5) is zero, so that $E(u)=E(v)$.

For each $b \in \mathcal{B}^{\text {reg }}$ there is a dense subset $\mathcal{J}^{\text {reg }}(b) \subset \mathcal{J}$ of almost complex structures $J$ such that all solutions of (4) with arbitrary $y_{-}, y_{+}$are regular, meaning that $D_{u}$ is onto. Then each $\mathcal{M}_{k}\left(y_{-}, y_{+}, b, J\right)$ has a natural structure of a smooth $k$-dimensional manifold. Moreover, the quotients $\mathcal{M}_{1}\left(y_{-}, y_{+}, b, J\right) / \mathbb{R}$ by translation in $s$-direction are actually finite sets. Denoting by $n\left(y_{-}, y_{+}\right)$the number of points mod 2 in each of them, one defines a differential $\partial_{b, J}: C F_{*}(\phi, b) \rightarrow C F_{* \pm 1}(\phi, b)$ by $\partial_{b, J}\left\langle y_{-}\right\rangle=$ $\sum_{y_{+}} n\left(y_{-}, y_{+}\right)\left\langle y_{+}\right\rangle$. This satisfies $\partial_{b, J} \circ \partial_{b, J}=0$, and its homology is by definition the Floer homology $H F_{*}(\phi)$. The necessary analysis is the same as in the slightly different situations considered in [1], [4]. In fact, the pertubation does not really affect the analytic arguments, since a change of variables transforms (4) into a similar equation without the $X_{b, t}$ term. Note also that bubbling is not an issue, as we have $\pi_{2}(M)=0$.

A continuation argument shows that Floer homology is independent of the choice of $b$ and $J$ up to canonical isomorphism. This involves an equation, with periodicity and convergence conditions as before,

$$
\frac{\partial u}{\partial s}+J_{s, t}(u)\left(\frac{\partial u}{\partial t}-X_{b, s, t}(u)\right)=0
$$

Here the $b_{s, t}$ are closed one-forms on $M$, which are $s$-independent if $|s|$ is sufficiently large; and one supposes again that $b_{s, t}=-\partial a_{s, t} / \partial t+d H_{s, t}$, where $a_{s, t}$ and $H_{s, t}$ are also $s$-independent for $|s| \gg 0$. The construction follows closely the familiar pattern [10] and only one step deserves a separate mention, namely, the proof of the energy estimate. Let $u$ be a solution of 
(6) with limits $y_{ \pm}$. A straightforward computation yields

$$
\begin{aligned}
E(u)=\int_{\mathbb{R} \times[0 ; 1]}\left|\frac{\partial u}{\partial s}\right|^{2}= & \int_{\mathbb{R} \times[0 ; 1]} \omega\left(\frac{\partial u}{\partial s}, \frac{\partial u}{\partial t}\right) \\
& -\int_{\{+\infty\} \times[0 ; 1]}\left(a_{s, t}\left(\frac{\partial u}{\partial t}\right)+H_{s, t}(u)\right) \\
& +\int_{\{-\infty\} \times[0 ; 1]}\left(a_{s, t}\left(\frac{\partial u}{\partial t}\right)+H_{s, t}(u)\right) \\
& +\int_{\mathbb{R} \times[0 ; 1]} \frac{\partial H_{s, t}}{\partial s}(u)+\int_{\mathbb{R} \times[0 ; 1]} \frac{\partial a_{s, t}}{\partial s}\left(X_{b, s, t}(u)\right) \\
& +\int_{\mathbb{R} \times[0 ; 1]} \frac{\partial a_{s, t}}{\partial s}\left(J_{s, t}(u) \frac{\partial u}{\partial s}\right) .
\end{aligned}
$$

One can show in the same way as in Lemma 9 that the first term $\int u^{*} \omega$ depends only on $y_{ \pm}$and on the expected dimension of the moduli space of solutions near $u$. The second and third term obviously depend only on $y_{ \pm}$; the fourth and fifth one are bounded by constants independent of $u$; and the last one by a constant times $E(u)^{1 / 2}$. It follows that on any relevant moduli space of solutions there is an inequality $E(u) \leq$ const $_{1}+$ const $_{2} E(u)^{1 / 2}$, giving an upper bound for $E(u)$.

Let $\left(\phi \circ \psi_{t}\right)_{0 \leq t \leq 1}$ be an isotopy in $\operatorname{Symp}^{m}(M, \omega)$ such that $\psi_{t}=\mathrm{id}_{M}$ for small $t$, and which is constant for $t$ near 1 . Let $c_{t}$ be the closed one-forms on $M$ which generate the isotopy, $c_{t}=\omega\left(\cdot, D \psi_{t}^{-1}\left(\partial \psi_{t} / \partial t\right)\right)$. Because of Lemma 6, the fact that the isotopy stays within $\operatorname{Symp}^{m}(M, \omega)$ means that

$$
\left[c_{t}\right] \in \operatorname{im}\left(\mathrm{id}-\phi^{*}\right) \subset H^{1}(M ; \mathbb{R}) .
$$

Set $\phi^{\prime}=\phi \circ \psi_{1}$ and define a diffeomorphism $\Psi: \Lambda_{\phi^{\prime}} \rightarrow \Lambda_{\phi}$ by $(\Psi y)(t)=$ $\psi_{t}(y(t))$ for $0 \leq t \leq 1$. A straightforward computation shows that $\Psi^{*} \alpha_{\phi, b}=$ $\alpha_{\phi^{\prime}, b^{\prime}}$ where $b_{t}^{\prime}=\psi_{t}^{*} b_{t}-c_{t}$ for $0 \leq t \leq 1$. (7) implies that $b^{\prime}=\left(b_{t}^{\prime}\right) \in \mathcal{B}$. Choosing $b \in \mathcal{B}^{\text {reg }}$ and $J \in \mathcal{J}^{\text {reg }}(b)$ and setting $J_{t}^{\prime}=\psi_{t}^{*} J_{t}$, one has that $b^{\prime} \in \mathcal{B}^{\text {reg }}$ and $J^{\prime} \in \mathcal{J}^{\text {reg }}\left(b^{\prime}\right)$. $\Psi$ induces an isomorphism of chain complexes $\left(C F_{*}\left(\phi^{\prime}, b^{\prime}\right), \partial_{b^{\prime}, J^{\prime}}\right) \rightarrow\left(C F_{*}(\phi, b), \partial_{b, J}\right)$, which implies that

$$
H F_{*}\left(\phi^{\prime}\right) \cong H F_{*}(\phi) \text {. }
$$

This, together with the first part of Lemma 7 , allows one to define $H F_{*}(g)$ for $g \in \Gamma$ as the Floer homology of any monotone symplectic representative. One can actually prove that the isomorphisms (8) are canonical, which means independent of the choice of the path $\left(\phi \circ \psi_{t}\right)$ between $\phi$ and $\phi^{\prime}$. We will not really use this, but it seems appropriate at least to outline the argument. Suppose first that we have an isotopy with $\psi_{1}=\mathrm{id}_{M}$, so that $\phi^{\prime}=\phi$. Then our construction defines an automorphism of $H F^{*}(\phi)$. In a slightly different situation, such automorphisms were studied in [12], and 
a suitably adapted version of [12, Proposition 5.1] shows that they depend only on the class of $\left(\psi_{t}\right)_{0 \leq t \leq 1}$ in $\pi_{1}\left(\operatorname{Symp}^{m}(M, \omega)\right)$. This fundamental group is trivial by Lemma 7, hence the automorphism of $H F^{*}(\phi)$ is the identity. The general statement can be derived from this special case, by considering the composition of a map (8) and the inverse of another one, coming from a different isotopy.

\section{Quantum cap product.}

Take $\phi \in \operatorname{Symp}^{m}(M, \omega), b \in \mathcal{B}^{\text {reg }}$ and $J \in \mathcal{J}^{\text {reg }}(b)$ as before. We begin by recalling the Gromov-Floer compactification $\overline{\mathcal{M}}_{k}\left(y_{-}, y_{+}, b, J\right)$ of the space $\mathcal{M}_{k}\left(y_{-}, y_{+}, b, J\right)$. As a set, this is the disjoint union of

$$
\begin{array}{r}
\prod_{i=1}^{p} \mathcal{M}_{l_{i}}\left(y_{-}^{i-1}, y_{-}^{i}, b, J\right) / \mathbb{R} \times \mathcal{M}_{k-\sum_{i} l_{i}-\sum_{j} m_{j}}\left(y_{-}^{p}, y_{+}^{0}, b, J\right) \\
\times \prod_{j=1}^{q} \mathcal{M}_{m_{j}}\left(y_{+}^{j-1}, y_{+}^{j}, b, J\right) / \mathbb{R}
\end{array}
$$

for all $p, q \geq 0, l_{i}, m_{j}>0$, and $y_{-}^{i}, y_{+}^{j} \in \mathcal{Z}(b)$ with $y_{-}^{0}=y_{-}$and $y_{+}^{q}=$ $y_{+}$. We will not write down the definition of the topology, but one of its properties is that if a sequence $\left(u_{k}\right)$ in $\mathcal{M}_{k}\left(y_{-}, y_{+}, b, J\right)$ has a limit $\left(\left[v_{1}^{-}\right], \ldots,\left[v_{p}^{-}\right], v,\left[v_{1}^{+}\right], \ldots,\left[v_{q}^{+}\right]\right)$then the $u_{k}$ converge uniformly on compact subsets towards the principal component $v$ (which may be $s$-independent). As a consequence, the evaluation map $\eta: \mathcal{M}_{k}\left(y_{-}, y_{+}, b, J\right) \rightarrow M, \eta(u)=$ $u(0,1)$, has a natural continuous extension $\bar{\eta}$ to the compactification.

Choose a Morse function $f$ on $M$ and a Riemannian metric for which $\nabla f$ is Morse-Smale. We may assume that the unstable (for the flow of $\nabla f$ ) manifolds $W^{u}\left(f, x_{-}\right) \subset M$ of all critical points $x_{-}$are transverse to $\eta$ for all $y_{-}, y_{+}$and $k$. If $k$ is equal to the Morse index $\mu\left(x_{-}\right)$, the preimage $\eta^{-1} W^{u}\left(f, x_{-}\right) \subset \mathcal{M}_{k}\left(y_{-}, y_{+}, b, J\right)$ is zero-dimensional. Moreover, a dimension count using (9) shows that the two sets

$$
\left.\begin{array}{c}
\bar{\eta}^{-1}\left(\overline{W^{u}\left(f, x_{-}\right)} \backslash W^{u}\left(f, x_{-}\right)\right) \\
\bar{\eta}^{-1} W^{u}\left(f, x_{-}\right) \backslash \eta^{-1} W^{u}\left(f, x_{-}\right)
\end{array}\right\} \subset \overline{\mathcal{M}}_{k}\left(y_{-}, y_{+}, b, J\right)
$$

are empty. This implies that $\eta^{-1} W^{u}\left(f, x_{-}\right) \subset \mathcal{M}_{k}\left(y_{-}, y_{+}, b, J\right)$ is closed inside the Gromov-Floer compactification, hence a finite set. Let $q\left(x_{-}, y_{-}, y_{+}\right)$ be the number of points mod 2 in it; one defines a homomorphism

$$
\begin{aligned}
& C M^{*}(f) \otimes C F_{*}(\phi, b) \longrightarrow C F_{*}(\phi, b), \\
& \left\langle x_{-}\right\rangle \otimes\left\langle y_{-}\right\rangle \longmapsto \sum_{y_{+}} q\left(x_{-}, y_{-}, y_{+}\right)\left\langle y_{+}\right\rangle .
\end{aligned}
$$


The next step is to consider the spaces $\eta^{-1} W^{u}\left(f, x_{-}\right) \subset \mathcal{M}_{k}\left(y_{-}, y_{+}, b, J\right)$ in the case where they are one-dimensional, which is when $k=\mu\left(x_{-}\right)+1$. A

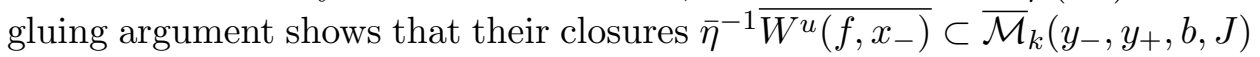
are one-dimensional manifolds with boundary, and by counting their boundary points one sees that

$$
\begin{aligned}
\sum_{x} l\left(x_{-}, x\right) q\left(x, y_{-}, y_{+}\right)+\sum_{y} n\left(y_{-}, y\right) q\left(x_{-}, y, y_{+}\right) & \\
& +\sum_{y} n\left(y, y_{+}\right) q\left(x_{-}, y_{-}, y\right)=0
\end{aligned}
$$

where $l$ and $n$ are defined in Sections 2c and 3, respectively. This means that (10) is a chain map. The induced map on homology is, by definition, the quantum cap product $*: H^{*}(M ; \mathbb{Z} / 2) \otimes H F_{*}(\phi) \rightarrow H F_{*}(\phi)$. This can be proved to be independent of the Morse function $f$ and the Riemannian metric, and of the choices made in the definition of $H F_{*}(\phi)$. One can also prove, but we will not really use it here, that it makes $H F_{*}(\phi)$ into a module over the cohomology ring of $M$. Moreover the quantum cap product commutes with the isomorphisms (8), so that it gives rise to a well-defined module structure on $H F_{*}(g)$ for $g \in \Gamma$. Details can be found in [5], [8], [11], [6]; the situation considered in those references tends to differ slightly from the one here, but the arguments carry over with minimal changes.

The construction of the refined product $\tilde{*}$ parallels that of $*$. Let $p_{\phi}$ : $\tilde{M}_{\phi} \rightarrow M$ be the covering introduced in Section 2 a. The evaluation maps $\eta$ have natural lifts $\xi$,

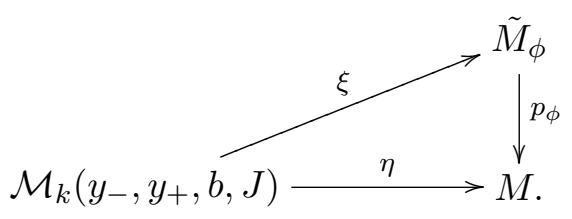

Namely, $\xi(u)$ is the pair $(x,[c])$ consisting of $x=u(0,1)$ and the homotopy class $[c]$ of the path $c(t)=u(0, t)$ from $\phi(x)=u(0,0)$ to $x$. In the same way as before, one sees that $\xi$ extends naturally to a continuous map $\bar{\xi}$ on the Gromov-Floer compactification, which is a lift of $\bar{\eta}$.

Consider the pullback Morse function $\tilde{f}=f \circ p_{\phi}$ and metric on $\tilde{M}_{\phi}$. Then $p_{\phi}^{-1} W^{u}\left(f, x_{-}\right)$is the disjoint union of $W^{u}\left(\tilde{f}, \tilde{x}_{-}\right)$over all preimages $\tilde{x}_{-}$of $x_{-}$. Together with the commutativity of (11) this yields that

$$
\eta^{-1} W^{u}\left(f, x_{-}\right)=\bigsqcup_{p_{\phi}\left(\tilde{x}_{-}\right)=x_{-}} \xi^{-1} W^{u}\left(\tilde{f}, \tilde{x}_{-}\right) \subset \mathcal{M}_{k}\left(y_{-}, y_{+}, b, J\right) .
$$

In particular, if $k$ equals the Morse index of $x_{-}$(and hence of $\tilde{x}_{-}$), the sets $\xi^{-1} W^{u}\left(\tilde{f}, \tilde{x}_{-}\right) \subset \mathcal{M}_{k}\left(y_{-}, y_{+}, b, J\right)$ are finite, and only a finite number of 
them is nonempty as one ranges over all $\tilde{x}_{-}$. Denoting by $\tilde{q}\left(\tilde{x}_{-}, y_{-}, y_{+}\right)$the number of points mod two in these sets, one defines

$$
\begin{aligned}
& C M^{*}(\tilde{f}) \otimes C F_{*}(\phi, b) \longrightarrow C F_{*}(\phi, b), \\
& \left\langle\tilde{x}_{-}\right\rangle \otimes\left\langle y_{-}\right\rangle \longmapsto \sum_{y_{+}} \tilde{q}\left(\tilde{x}_{-}, y_{-}, y_{+}\right)\left\langle y_{+}\right\rangle .
\end{aligned}
$$

From looking at the one-dimensional spaces $\eta^{-1} W^{u}\left(\tilde{f}, \tilde{x}_{-}\right)$and applying to their ends the same argument as before, one gets

$$
\begin{aligned}
\sum_{\tilde{x}} \tilde{l}\left(\tilde{x}_{-}, \tilde{x}\right) \tilde{q}\left(\tilde{x}, y_{-}, y_{+}\right)+\sum_{y} n\left(y_{-}, y\right) \tilde{q}\left(\tilde{x}_{-}, y, y_{+}\right) & \\
& +\sum_{y} n\left(y, y_{+}\right) \tilde{q}\left(\tilde{x}_{-}, y_{-}, y\right)=0
\end{aligned}
$$

with $\tilde{l}$ as defined in Section 2c, so that $\tilde{*}$ is a chain homomorphism. Moreover, by definition $\sum_{p_{\phi}\left(\tilde{x}_{-}\right)=x_{-}} \tilde{q}\left(\tilde{x}_{-}, y_{-}, y_{+}\right)=q\left(x_{-}, y_{-}, y_{+}\right)$, so that the maps (10) and (12) fit into a commutative diagram

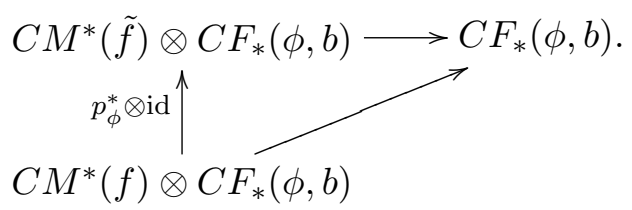

Therefore (12) induces a map $\tilde{*}: H^{*}\left(\tilde{M}_{\phi}\right) \otimes H F_{*}(\phi) \rightarrow H F_{*}(\phi)$ which satisfies the conditions of Lemma 3 . Having that, we can now prove the two theorems stated at the beginning of the paper. If $g=[\phi]$ is nontrivial, $H^{2}\left(\tilde{M}_{\phi} ; \mathbb{Z} / 2\right)$ is zero by Lemma 5 , which means that $a * y=p_{\phi}^{*}(a) \tilde{*} y=0$ for $a \in H^{2}(M ; \mathbb{Z} / 2)$ and $y \in H F_{*}(g)$, as claimed in Theorem 1 . In the same way, Lemma 3 and Lemma 4 imply Theorem 2.

Acknowledgments. The importance of the quantum module structure for symplectic isotopy questions was first pointed out by Callahan. Lemma 3 is inspired by a construction of Viterbo [13]. Ivan Smith and Dietmar Salamon made useful comments on a preliminary version of this paper.

\section{References}

[1] S. Dostoglou and D. Salamon, Self-dual instantons and holomorphic curves, Annals of Math., 139 (1994), 581-640, MR 95g:58050, Zbl 0812.58031.

[2] C.J. Earle and J. Eells, The diffeomorphism group of a compact Riemann surface, Bull. Amer. Math. Soc., 73 (1967), 557-559, MR 35 \#3705, Zbl 0196.09402.

[3] A. Fathi, F. Laudenbach and V. Poénaru, in 'Travaux de Thurston sur les surfaces', Astérisque, 66-67, Soc. Math. France, 1979, MR 82m:57003. 
[4] A. Floer, H. Hofer and D. Salamon, Transversality in elliptic Morse theory for the symplectic action, Duke Math. J., 80 (1995), 251-292, MR 96h:58024, Zbl 0846.58025.

[5] Le Hong Van and K. Ono, Cup-length estimate for symplectic fixed points, in 'Contact and symplectic geometry' (C.B. Thomas, ed.), Cambridge Univ. Press, (1996), 268295, MR 97m:58078, Zbl 0874.53030.

[6] G. Liu and G. Tian, On the equivalence of multiplicative structures in Floer homology and quantum homology, Acta Math. Sin. (Engl. Ser.), 15 (1999), 53-80, MR 2001g:53154, Zbl 0928.53041.

[7] J. Moser, On the volume elements on a manifold, Trans. Amer. Math. Soc., 120 (1965), 286-294, MR 32 \#409, Zbl 0141.19407.

[8] S. Piunikhin, D. Salamon and M. Schwarz, Symplectic Floer-Donaldson theory and quantum cohomology, in 'Contact and symplectic geometry' (C.B. Thomas, ed.), Cambridge Univ. Press, (1996), 171-200, MR 97m:57053, Zbl 0874.53031.

[9] Y. Ruan and G. Tian, Bott-type symplectic Floer cohomology and its multiplication structures, Math. Res. Lett., 2 (1995), 203-219, MR 96a:57074, Zbl 0844.57023.

[10] D. Salamon and E. Zehnder, Morse theory for periodic solutions of Hamiltonian systems and the Maslov index, Comm. Pure Appl. Math., 45 (1992), 1303-1360, MR 93g:58028, Zbl 0766.58023.

[11] M. Schwarz, Equivalences for Morse homology, in 'Geometry and topology in dynamics' (M. Barge and K. Kuperberg, eds.), Contemp. Math., 246, Amer. Math. Soc., (1999), 197-216, MR 2000j:57070, Zbl 0951.55009.

[12] P. Seidel, $\pi_{1}$ of Hamiltonian automorphism groups and invertibles in quantum homology rings, Geom. Funct. Anal., 7 (1997), 1046-1095, MR 99b:57068, Zbl 0928.53042.

[13] C. Viterbo, The cup-product on the Thom-Smale-Witten complex, and Floer cohomology, in 'The Floer memorial volume' (H. Hofer, C. Taubes, A. Weinstein, and E. Zehnder, eds.), Birkhäuser, (1995), 609-625, MR 96k:58038, Zbl 0843.57031.

Received November 6, 2000 and revised March 6, 2001.

\section{School of Mathematics}

Institute of Advanced Study

PRINCETON, NJ 08540

E-mail address: seidel@math.ias.edu 